\title{
Simulation Environment for Development of Unmanned Helicopter Automatic Take-off and Landing on Ship Deck
}

\author{
Antonio Vitale $^{1} \quad$ Davide Bianco $^{1} \quad$ Gianluca Corraro $^{1} \quad$ Angelo Martone $^{1} \quad$ Federico Corraro $^{1}$ \\ Alfredo Giuliano ${ }^{2}$ Adriano Arcadipane ${ }^{2}$ \\ ${ }^{1}$ On-boar Systems and ATM Department, CIRA - Italian Aerospace Research Centre, Capua (CE), Italy, \{a.vitale, \\ d.bianco, g.corraro, a.martone, f.corraro\} @cira.it \\ ${ }^{2}$ Electrical and Avionics Systems Department, Finmeccanica - Helicopter Division, Cascina Costa (VA), Italy, \\ \{Alfredo.Giuliano, Adriano.Arcadipane\} @finmeccanica.com
}

\begin{abstract}
Helicopter take-off and landing operations on ship carrier are very hazardous and training intensive. Guidance, Navigation and Control algorithms can help pilots to face these tasks by significantly reducing the workload and improving safety level. Anyway, the design and verification of such algorithms require the availability of suitable simulation environments that shall be a trade-off between simplicity and accuracy. This paper presents the simulation models developed to support the design, pre-flight verification and validation of helicopter trajectory generation and tracking algorithms for automated take-off and landing on a frigate deck. The process for generation and testing of the code to be integrated into the real-time Software-Inthe-Loop simulator is also described. Such fast time and real-time simulation environments contributed to reduce algorithms design time, risks and costs, by limiting the required flight test activities. Take-off and landing algorithms developed by using the proposed simulation environments were successfully demonstrated in flight.
\end{abstract}

Keywords: GNC, helicopter, sensor, ship, turbulence

\section{Introduction}

Vertical take-off and landing operations of aerial vehicle on ship's deck enhance mission capabilities for military and civilian users. Anyway, these operations are the most dangerous flight phases for helicopters (Padfield, 1998; Lee, 2005). Indeed, a pilot have to deal with an invisible ship air wake, poor visible cueing and a landing spot which is heaving, rolling, pitching and yawing. At the same time the pilot shall also monitor vehicle's structural, aerodynamic and control limits. Moreover, operations take place in close proximity to the superstructure of the ship, that means there is little margin for error and the consequences of a significant loss of positional accuracy by the pilot can be severe.

The availability of Guidance Navigation and Control (GNC) algorithms for automatic operations can help pilots to face these tasks by significantly reducing operator workload, improving safety level and flight handling qualities. To develop these algorithms, suitable simulation environments are essential in order to reduce the flight test time and cost and to establish safe operating envelopes. The simulation tools shall be able to model all the relevant phenomena, such as helicopter flight dynamics (including on board sensors and actuators), the motion of the ship for the given sea state, the influence on the helicopter of the ship air wake and of the environment in general.

It is worth to note that modelling and simulation of each of the above listed phenomena is not a trivial task. Indeed, the simulation of the helicopter flight behavior includes kinematics, dynamics and aerodynamics of its subsystems (main rotor, fuselage, empennage, tail rotor, power plant, primary flight control system, on board sensors).

The vehicle's equations of motion, even if presented in several textbooks (Padfield, 1996; Johnson, 1994), are differential high order, nonlinear, coupled, and contain a large number of parameters, which often cannot be directly measured (Tishler et al., 2006). On the other hand, simplified models, which are able to catch the relevant dynamics, are typically required for GNC design purpose (Lee, 2005), to enhance physical understanding and lower the computational load. To this aim, linear parametrized models have been widely used (Tishler et al., 2006), but they are inadequate for accurate simulation of the vehicle dynamics when state variables significantly deviate from the linearization point (Gavrilets, 2006). Therefore, a suitable trade-off between model complexity and simulation accuracy shall be performed.

Another relevant topic concerns ship motion, which is an important issue for helicopter deck operations. For helicopter GNC algorithms design and analysis purpose, ship motion is usually represented through linear models or simplified nonlinear models with benign nonlinearities to capture the essential behavior of the vessel ( $\mathrm{Li}, 2009)$. Ship motion can also be modelled using pre-computed or measured time histories (Carico et al., 2003). In any case, the ship model shall take into account the effect of the environment, and, in particular, 
of the sea waves (Perez, 2005), which lead an undesirable low frequency disturbance into the motion of the vessel.

Finally yet importantly, the ship produces an air wake, which affects the helicopter dynamics. Indeed, ship air wake contains large velocity gradients and area of turbulence, generated by complex mechanisms of vortex dynamics near the ship deck, which greatly impair controllability of the flying vehicle and require additional control efforts to avoid accidents and to compensate abrupt changes in thrust level. Several accurate and complex CFD models of ship air wake are proposed in the literature (Kääriä, 2012), but CFD simulations produce a large amount of data and their use for GNC design and real time testing is usually unfeasible (Lee, 2005).

Stochastic turbulence models have been also proposed to represent the air wake with reasonable accuracy (Lee, 2005; Yang et al., 2009). These models may provide some insight into the effects of the air wake that are typically enough relevant for real-time simulations and flight control systems design.

It is also worth to note that, with reference to all the discussed models, a suitable code generation procedure and testing methodology shall be defined, in order to generate reliable real-time simulation models, applicable for GNC algorithms verification and performance assessment.

This paper presents the simulation environment developed by the Italian Aerospace Research Centre and Finmeccanica in order to support the design and verification of algorithms for helicopter trajectory generation and tracking, during an automated take-off and landing on a frigate deck. Matlab/Simulink was used for implementing such simulation environment, which constitutes an alternative to the already existing Finmeccanica GNC validation environment.

The proposed models, although simplified, are able to take into account the main effects of the sea's disturbance on the ship motion and of the ship air wake on the helicopter trajectory. Concerning the helicopter vehicle dynamics, its model emulates the relevant closed loop performance of the vehicle and includes operating envelope limitations through a model for aerodynamic forces and thrust computation, whose parameters are identified from experimental data.

The paper also includes some fast time simulation results compared to experimental data, demonstrating that the proposed simulation environment is accurate enough for GNC algorithms design.

Finally, some models of the above mentioned simulation environment were also integrated into the detailed Software-in-the-Loop Simulator of Finmeccanica, to perform real-time verification and validation of the whole Flight Management System (FMS). Therefore, a real-time automatic code generation process has been defined and implemented, in order to keep consistency between the simulation environment used for design, and the one used for final software verification. The paper briefly describes such generation process, which allowed producing reliable software code, compliant to DO-178C standard and Finmeccanica own implementation rules.

The proposed fast time simulation environment dramatically reduced the algorithms design time, risks and costs, by limiting the required flight test activities.

The take-off and landing algorithms developed by using the simulation environment described in this paper were successfully demonstrated in flight, by means of a full-size optionally piloted helicopter: the Finmeccanica SW-4 SOLO.

\section{Simulation Models}

The model based design process of a Guidance Navigation and Control system requires the development of simulation models with different complexity level, to be used in the various development phases, as shown in Figure 1.

The present section describes the mathematical models integrated into the simulation environment that was employed to design the helicopter trajectory generation and tracking algorithms for automated takeoff and landing on a frigate deck. Figure 2 shows the functional architecture of such environment.

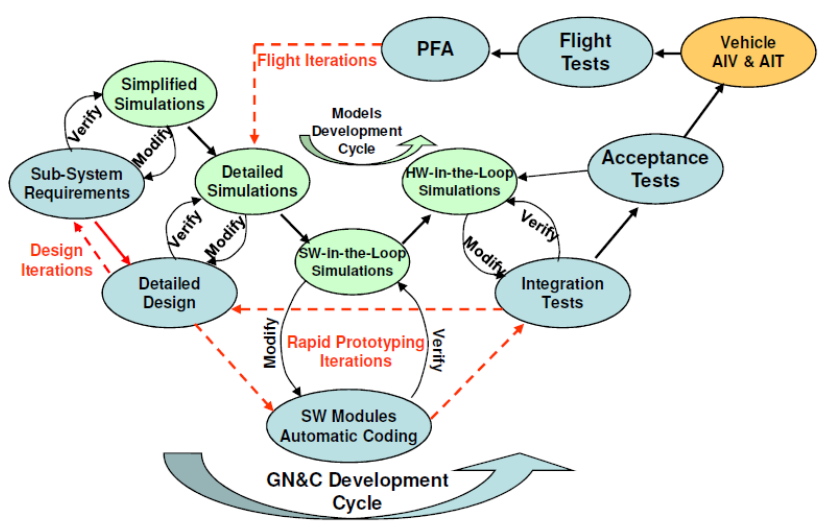

Figure 1. GNC Technology Development Cycle.

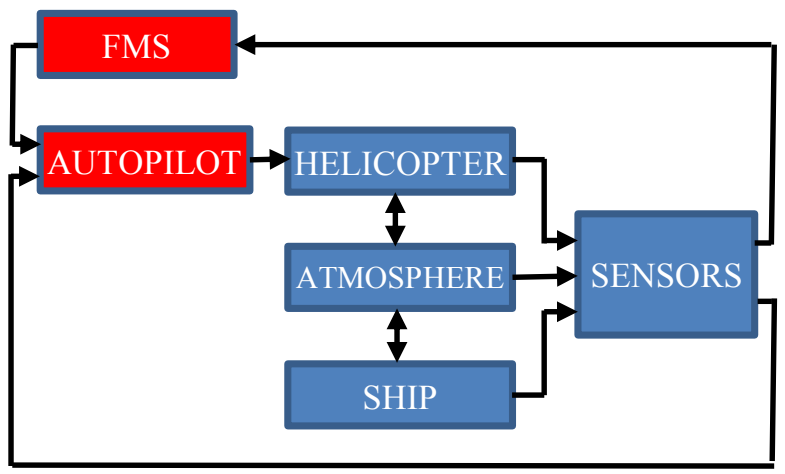

Figure 2. Simulation Environment functional architecture. 
The blue blocks represent the simulation models, scope of this paper, whereas the red blocks are the GNC algorithms. The following sub-sections describe in detail each blue block.

\subsection{Helicopter Model}

Trajectory generation and tracking algorithms typically require the knowledge of vehicle's position and velocity only. Therefore, for the design and preliminary verification of such algorithms, it is sufficient and cost effective to model only the closed loop attitude dynamic response of the vehicle coupled with the high-level modes of the autopilot system. With this approach, a rigid body with three degrees of freedom, subject to external forces, and the rotational dynamic response of the vehicle to the autopilot commands represent the helicopter dynamics.

While this modelling approach is widely used in fixed-wing aircraft for guidance algorithms design, it is quite unusual for helicopters, because it does not take into account the coupled dynamics of the rotor flexibility with the helicopter rigid flight mechanics (Tishler et al., 2006).

Key original contribution of this paper is the development of a mixed empirical and physical formulation of the equations, so that the resulting simulation model includes only the low frequency effects of the neglected helicopter dynamics. As demonstrated by the comparisons with flight data reported in this paper, this allows obtaining enough accurate simulation results during the quasi-static manoeuvers of take-off and landing, while still taking into account the disturbance effects of wind and ship air wake.

The model assumes flat and fixed Earth, with constant gravity acceleration, and quasi-stationary variation of the vehicle mass (only due to fuel consumption).

The model's commands are the reference attitude and collective, while wind velocity $\left(\underline{\mathrm{V}}_{\mathrm{w}}\right)$ is the disturbance input.

The actual attitude $\left(\varphi_{\mathrm{H}}, \vartheta_{\mathrm{H}}, \psi_{\mathrm{H}}\right)$ and collective $\left(\delta_{\text {coll }}\right)$ of the vehicle, used in (1) for computation of forces, are modelled by unitary gain second order filters applied to the commands provided as input to the helicopter model. Such filtered Euler angles and collective and their rates are also saturated to account for actuator velocity limitations and some inner autopilot protection functions. Overall, the linear filters and related saturations model the closed loop performance of the inner autopilot modes. The parameters of both these filters and saturations are scheduled with respect to airspeed and they were identified by analyzing flight data gathered in specific manoeuvers.

The outputs are the helicopter position, velocity, load factors, actual attitude and angular rates.
The following equations of motion of the vehicle centre of mass (CoM), in North-East-Down (NED) inertial reference frame (McCormick, 1995), compute such outputs:

$$
\begin{gathered}
m \underline{\dot{\mathrm{V}}}=\underline{\mathrm{F}}\left(\varphi_{\mathrm{H}}, \vartheta_{\mathrm{H}}, \psi_{\mathrm{H}}, \delta_{\text {coll }}, \underline{\mathrm{V}}_{\mathrm{W}}\right) \\
\dot{\mathrm{P}}_{\mathrm{H}}=\underline{\mathrm{V}}_{\mathrm{H}} \\
\dot{h}=-w
\end{gathered}
$$

where $\mathrm{V}$ is the inertial velocity vector, $\underline{\mathrm{V}}_{\mathrm{H}}$ and $w$ are its horizontal (included into the North-East plane) and vertical components (positive down), respectively; $\underline{P}_{H}$ is the horizontal position and $h$ the altitude of the vehicle $\mathrm{CoM} ; m$ is the helicopter mass and $\underline{\mathrm{F}}$ is the resultant force vector acting on the vehicle.

The force vector $\underline{F}$ is composed by gravitational force $\underline{\mathrm{W}}$ (constant, and directed along the down axis of the NED reference frame), aerodynamic force $\underline{F}_{A}$ and propulsive force $\underline{T}$.

The computation of aerodynamic and thrust forces is first performed in the vehicle body reference frame, and then it is rotated in NED reference frame. The aerodynamic forces in body axes $\left(F_{A i}^{B}\right)$ are as follows:

$$
\begin{gathered}
F_{A i}^{B}=q_{d y n} \sum_{\mathrm{j}} S_{j} c_{i, j}(\alpha, \beta) \\
V_{T A S}^{2}=\left(\underline{\mathrm{V}}-\underline{\mathrm{V}}_{\mathrm{W}}\right)^{T} \cdot\left(\underline{\mathrm{V}}-\underline{\mathrm{V}}_{\mathrm{W}}\right) \\
q_{d y n}=0.5 \rho \cdot V_{T A S}^{2} \\
\alpha=\operatorname{tg}^{-1}\left(w_{T A S} / u_{T A S}\right) \\
\beta=\sin ^{-1}\left(v_{T A S} / V_{T A S}\right)
\end{gathered}
$$

where $q_{d y n}$ is the dynamic pressure, $\rho$ is the air density, $V_{T A S} \equiv\left(u_{T A S}, v_{T A S}, w_{T A S}\right)$ is the helicopter true airspeed, $S_{j}$ is the reference aerodynamic surface of the j-th aerodynamic component (that is, fuselage, vertical and horizontal stabilizers) and $c_{i, j}$ the corresponding aerodynamic non-dimensional coefficient, which depends on the angle of attack $\alpha$ and sideslip angle $\beta$. Tabled functions express the aerodynamic coefficients using data extrapolated from flight experiments.

It is worthy to note that the aerodynamic angles $\alpha$ and $\beta$ are not defined when the helicopter airspeed is null, for example in hover condition with null wind speed. In this case, the aerodynamic forces are negligible and the aerodynamic angles are not computed.

The propulsive force is evaluated by using the following semi-empirical linear model (Gavrilets, 2003):

$$
T=z_{\text {coll }}\left(V_{T A S}\right) \cdot \delta_{\text {coll }}+z_{w}\left(V_{T A S}\right) \cdot w
$$

The parameter $z_{\text {coll }}$ is a gain between the thrust and the collective command $\delta_{\text {coll }}$ in level flight trim conditions. It is scheduled as a function of the forward speed of the aircraft with respect to air, and its values were identified applying a best-fit procedure of the rotor thrust data in 
different flight conditions provided by the helicopter manufacturer.

The parameter $z_{w}$ relates the thrust to the vertical speed. Although an analytical relation exists to express these parameters as function of vehicle characteristics (Gavrilets, 2003), in the present work, $z_{w}$ was computed by fitting experimental data in climb and descent flight, and it is expressed as fraction of $z_{\text {coll }}$.

The thrust vector is assumed to point in the opposite direction of the body Z-axis. This hypothesis allows reproducing in simulation the trim values of pitch angle experimented in flight by the vehicle in level flight conditions.

\subsection{Atmosphere Model}

This model is in charge to reproduce the environmental conditions, in which the helicopter flies, that can influence the vehicle behaviour.

The model includes computation of atmospheric parameters (air density and temperature, static and dynamic pressure), wind velocity (wind shear, wind gust, atmospheric turbulence), and ship air wake experimented by the helicopter, based on its current position and velocity. International Standard Atmosphere (McCormick, 1995), von Karman model (von Karman, 1948) and standard wind model (MIL-F$8785 \mathrm{C}, 1991)$ are used for atmospheric parameters, turbulence and wind shear and gust, respectively.

Another element of originality included in this paper concerns the simplified ship air wake model, which is implemented as a stochastic phenomenon through a parameter modification of the von Karman turbulence model (von Karman, 1948).

In this model, independent white noise processes are suitably filtered to yield the desired forms of output power spectral density. The transfer functions $\left(X_{u g}, X_{v g}\right.$, $\left.X_{w g}\right)$ of these linear filters in the Laplace domain are:

$$
\begin{aligned}
& X_{u g}(s)= \\
& \sigma_{u} \sqrt{\left(2 \cdot L_{u}\right) /\left(\pi \cdot V_{T A S}\right)} \cdot \frac{1}{1+\left(L_{u} / V_{T A S}\right) \cdot s} \\
& X_{v g}(s)= \\
& \sigma_{v} \sqrt{\left(2 \cdot L_{v}\right) /\left(\pi \cdot V_{T A S}\right)} \cdot \frac{1+2 \sqrt{3} \cdot\left(L_{v} / V_{T A S}\right) \cdot s}{\left[1+\left(2 \cdot L_{v}\right) / V_{T A S} \cdot s\right]^{2}} \\
& X_{w g}(s)= \\
& \sigma_{w} \sqrt{\left(2 \cdot L_{w}\right) /\left(\pi \cdot V_{T A S}\right)} \cdot \frac{1+2 \sqrt{3} \cdot\left(L_{w} / V_{T A S}\right) \cdot s}{\left[1+\left(2 \cdot L_{w}\right) / V_{T A S} \cdot s\right]^{2}}
\end{aligned}
$$

where $\sigma_{\mathrm{u}}, \sigma_{\mathrm{v}}, \sigma_{\mathrm{w}}$ and $L_{u}, L_{v}, L_{w}$ are gains and scale factors, respectively, to be tuned through the analysis of CFD or experimental (wind tunnel or flight test) data.

Anyway, due to the unavailability of these data, such model parameters and their dependencies from helicopter state variables were determined through literature analysis and physical considerations.
The scale factors are set proportional to the characteristic lengths of the ship super-structure, which generates the wake. Since the effects of the wake on helicopter depend also from ship-helicopter relative position, the filters gains varied linearly with the ratio between ship speed and the square of the helicopter-ship distance.

Moreover, to take into account the local effect of the air ship wake disturbance and its dependence on wind direction, the wake's perturbation is only active within a limited size parallelepiped, which is oriented parallel to the wind speed and has width equal to the section of the super-structure orthogonal to the wind direction, length equal to three times the superstructure's section parallel to the wind direction, and height equal to three times the superstructure's height.

\subsection{Ship Model}

The ship translational motion is represented through kinematic relations, for the computation of undisturbed centre of mass position and velocity, plus an additive stochastic model, which simulates the sea wave disturbance on the ship. The applied equations for nominal position and velocity computation are:

$$
\begin{gathered}
\dot{\mathrm{V}}_{N o}=\left[\begin{array}{lll}
a_{x} & a_{y} & 0
\end{array}\right]^{T} \\
\underline{\mathrm{P}}_{N o}=\left[\begin{array}{lll}
u_{N o} & v_{N o} & -w_{N o}
\end{array}\right]^{T}
\end{gathered}
$$

where $a_{x}$ and $a_{y}$ are the commanded horizontal acceleration of the ship; $\underline{\mathrm{V}}_{N o} \equiv\left(u_{N o}, v_{N o}, w_{N o}\right)$ and $\underline{\mathrm{P}}_{N o} \equiv$ $\left(x_{N o}, y_{N o}, h_{N o}\right)$ are nominal velocity and position in NED reference frame, respectively. The actual position $\underline{\mathrm{P}}_{N} \equiv$ $\left(u_{N}, v_{N}, w_{N}\right)$ and velocity $\underline{\mathrm{V}}_{N} \equiv\left(x_{N}, y_{N}, h_{N}\right)$ are calculated by adding the sea disturbance $\underline{\eta} \equiv\left(\eta_{\mathrm{x}}, \eta_{\mathrm{y}}, \eta_{\mathrm{z}}\right)$ to nominal values:

$$
\begin{aligned}
& \underline{\mathrm{V}}_{N}= {\left[\begin{array}{lll}
u_{N o} & v_{N o} & 0
\end{array}\right]^{T}+\underline{\dot{\eta}} } \\
& \\
& \underline{\mathbf{P}}_{N}=\underline{\mathbf{P}}_{N o}+\underline{\eta}
\end{aligned}
$$

The attitude equations are defined independently as follows:

$$
\left[\begin{array}{c}
\varphi_{\mathrm{N}} \\
\theta_{\mathrm{N}} \\
\psi_{\mathrm{N}}
\end{array}\right]=\left[\begin{array}{c}
\varphi_{0} \\
\theta_{0} \\
\psi_{0}
\end{array}\right]+\left[\begin{array}{l}
\eta_{\varphi} \\
\eta_{\theta} \\
\eta_{\psi}
\end{array}\right]
$$

It is assumed that the ship does not steer when helicopter is close, therefore its Euler angles $\left(\varphi_{\mathrm{N}}, \theta_{\mathrm{N}}, \psi_{\mathrm{N}}\right)$ only depend on their initial values and on the sea disturbance $\left(\eta_{\varphi}, \eta_{\theta}, \eta_{\psi}\right)$.

The stochastic variables introduced in (16) and (17) for representing the sea disturbance are generated using the same equations. A mean velocity $V_{S}$ and displacement $D_{S}$ produced by the disturbance is associated to each variable. $V_{S}$ and $D_{S}$ depend on ship speed and sea state, and are provided by look up tables, which collect experimental data.

The sea disturbance is periodic and it pulsation $\omega$ is given by (Holthuijsen, 2017) 


$$
\omega=2 \pi V_{S} / D_{S}
$$

The time evolution of the generic component of the sea disturbance $\eta_{\mathrm{i}}$ is then evaluated as follows

$$
\eta_{\mathrm{i}}(t)=A_{i} \sin \left(\omega_{\mathrm{i}} t\right)
$$

The gain $A_{i}$ is a random variable with Rayleigh distribution, whose parameters depend on sea state and ship speed and are provided by a look up table based on experimental observations. During a simulation, the gain $A_{i}$ is updated at the end of each wave period (that is, each $D_{S} / V_{S}$ seconds) by performing a new random draw.

\subsection{Sensor Model}

Two kinds of sensors are available on-board the helicopter and are included into the simulation environment: a standard navigation suite (composed of an inertial navigation system and an air data system) and a differential GPS, with centimetric precision, denoted as Precision Positioning System (PPS) and needed for accurate relative navigation during take-off and landing operations.

Each measurement $(M)$ is computed starting from its simulated true value $(\bar{M})$ taken from the models of the helicopter, the ship or the atmosphere.

For what concerns the inertial navigation sensor, each true variable to be measured is filtered and sampled. Then it is corrupted by introducing a scale factor deviation $\left(C_{S F}\right)$, a bias $\left(e_{\text {bias }}\right)$, white noise $\left(e_{w h i t e}\right)$ and an additive magnetic declination error $\left(e_{d e c}\right)$, which is zero for all the measurements but the helicopter heading:

$$
M=C_{S F} \bar{M}+e_{\text {bias }}+e_{\text {white }}+e_{\text {dec }}
$$

The air data measurements are generated through the relation:

$$
M=\bar{M}+e_{\text {bias }}+e_{\text {white }}
$$

Concerning the GPS sensor, it is simulated corrupting the true measurements with bias, white noise and diluition of precision error $\left(e_{D O P}\right)$ :

$$
M=\bar{M}+e_{\text {bias }}+e_{\text {white }}+e_{D O P}
$$

All the additive errors in (20), (21) and (22) are stochastic and derived from the specification data sheet of the real sensor.

In the GPS model, these errors depend on the configuration of the sensor, which can work in SPS (Standard Positioning Service), DGPS (Differential GPS) and RTK (Real Time Kinematic) mode. The model also allows injecting a failure which degrades the precision of the sensor from RTK mode (also denoted as Precision Positioning System) to SPS mode.

\section{Code Generation and Verification}

As said, some of the developed models (e.g. ship, ship air wake and GPS sensor) were automatically software coded after the implementation in Matlab/Simulink, in order to allow their integration into the Finmeccanica real-time Software-In-the-Loop (SIL) simulator, which is used to test on ground the GNC prototype. Figure 3 shows the applied code generation process and testing methodology.

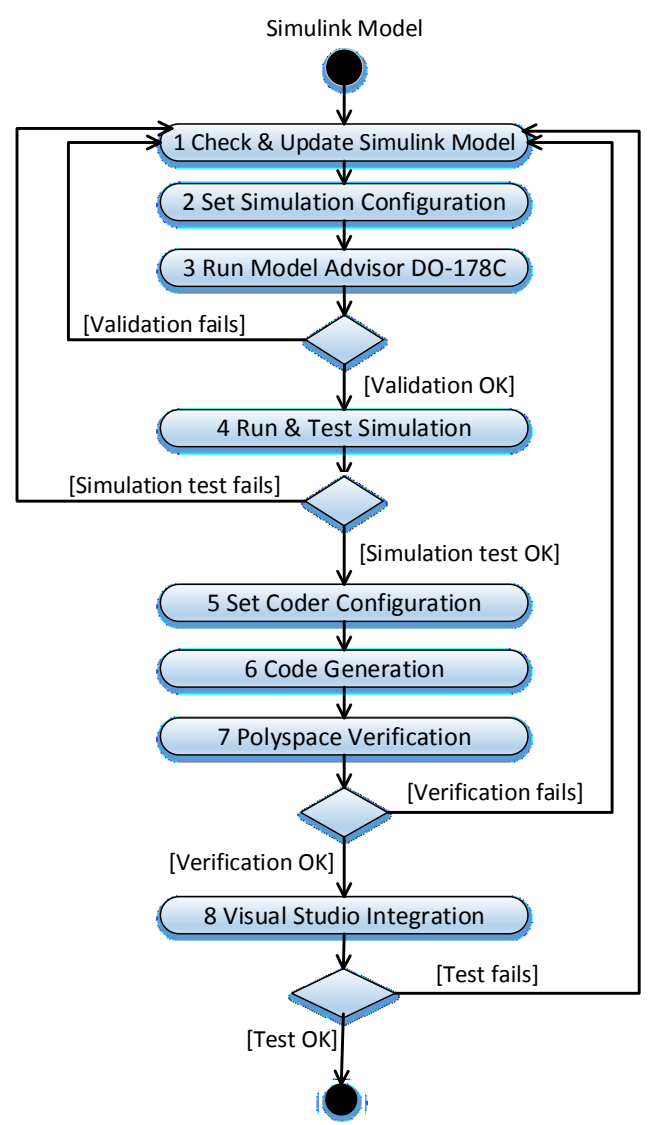

Figure 3. Code generation operational flow.

The flow starts with the selection of the Simulink model from which the code shall be generated. This model shall follow Finmeccanica proprietary design rules and specifications; to this end, a proprietary Simulink library have been developed and used to implement the models.

In step 1, the Model Update command in Simulink environment allows to check for errors and warnings. Then, the configuration settings are applied by running a Matlab script (step 2), that is customized to make the Simulink model compliant to the DO-178C standard. This compliance is verified in step 3 by means of the Mathworks Model Advisor tool. Next, the unit test for each Simulink model is performed, still in Simulink environment (step 4). In step 5, a proprietary Matlab script defines the Code Configuration settings; then the source C Code of the model is automatically generated (step 6) using Real Time Workshop. The Mathworks Polyspace tool is applied in step 7, to perform a static analysis of the generated code in order to check the absence of overflow, divide by zero, out of bounds array access, and other kind of run-time errors. If the generated code passes Polyspace tests, it can be integrated into Microsoft Visual Studio Environment 
(step 8) to be tested with the same test vectors used in Step 4. Finally, the test outputs of step 4 and step 8 are compared, in order to check the correctness of the generated code.

After that, the model code can be integrated into the final detailed simulation model, being sure that it performs exactly as the simulation environment used for design.

\section{Simulation Results}

The principal phenomena that influenced the design of the trajectories and tuning of the tracking algorithm for automatic take-off and landing are the wake phenomenon near the ship, the PPS availability along the trajectory, the disturbance of the sea waves on the ship deck motion and the performance and dynamic behavior of the helicopter.

The validity of the proposed helicopter model for GNC algorithm design can be demonstrated by Figure 4 where comparison of flight data versus simulation data is reported for attitude.

The differences that can be noted have negligible effects on the algorithm design and preliminary testing, as the helicopter low frequency behavior is almost accurately predicted. Similar results hold for acceleration, not reported here for the sake of brevity.

Moreover, Figure 5 compares the collective deflections in trim condition at $650 \mathrm{ft}$ altitude computed by using the model with a validation data set provided by Finmeccanica: the model reproduces quite well the vehicle behavior, confirming the validity of the proposed helicopter thrust model.
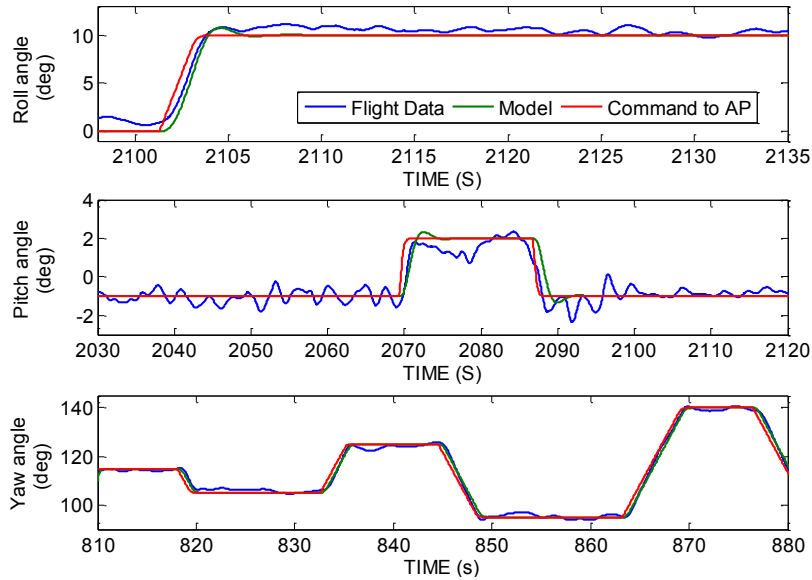

Figure 4. Comparison between simulated and experimental attitudes.

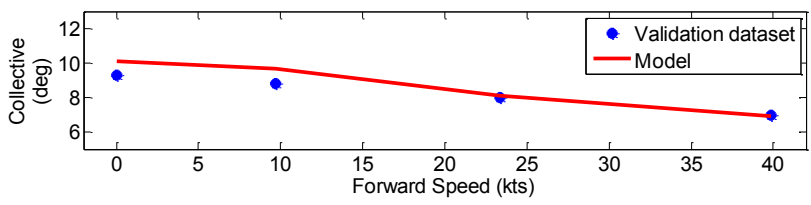

Figure 5. Comparison between simulated collective deflections in trim condition and validation data.

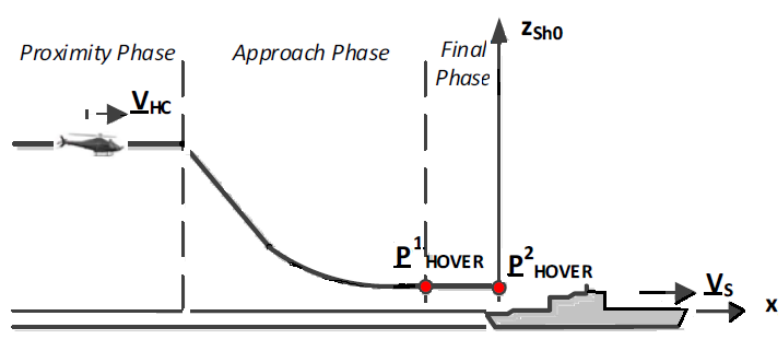

Figure 6. Schematic representation of the landing trajectory.

The other main simulated effects on a sample automatic landing trajectory are also presented below.

The designed landing trajectory, schematically shown in Figure 6, is structured in three phases. In the Proximity phase the helicopter is almost aligned with the ship direction at a desired speed in order to follow properly the descending path to the first relative hovering way point (Approach phase). In the Final phase, after the operator acknowledgment, the helicopter moves to the second relative hovering waypoint $\left(\underline{\mathrm{P}}^{2}\right.$ HOVER $)$ and finally lands on the ship deck.

The modelled action of the air wake on the helicopter vertical acceleration, during the automatic landing manoeuvers, is shown in Figure 7.

It is worth to note, in the second graph, how the effect of the air wake is null until the helicopter enters in a proper area (near $\underline{\mathrm{P}}^{1}{ }_{\text {HOVER }}$ ). As said, such area depends on the ship super-structure and wind direction (which in the test is aligned with the ship speed). When the ground operator gives the acknowledge command, the relative distance between the ship and the helicopter decreases while the wake effect increases. The same happens as the relative altitude decreases in the last manoeuver for deck landing.

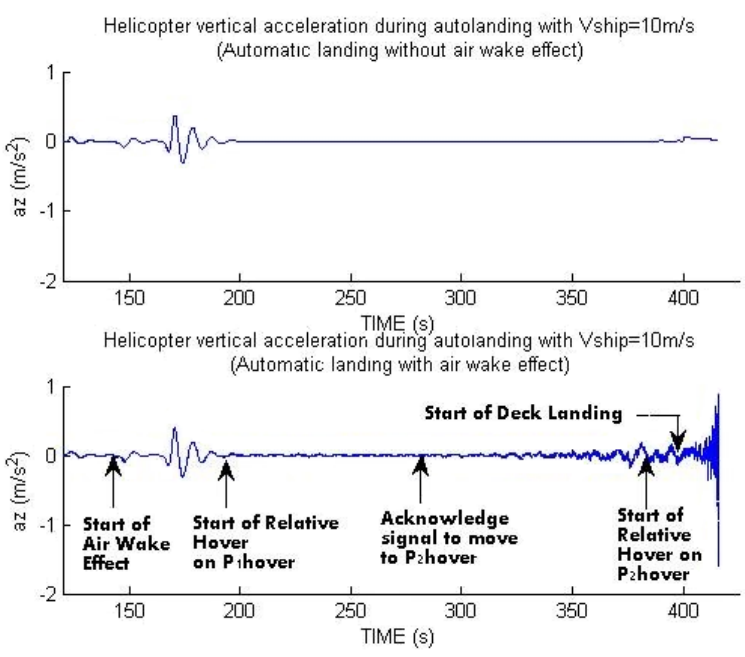

Figure 7. Air wake effect on the helicopter vertical acceleration.

Figure 8 presents the effect of the sea waves on the ship. It refers to Type 23 frigate at two different speeds for see state level six. 
As expected, it is highlighted how the frequency and the mean amplitude of ship deck motion decrease with the increase of the ship speed. Indeed wave disturbances are low pass filtered by the ship inertia, and the cut off frequency of this filter decreases when ship speed increases. Such movements are taken into account in the last part of the landing manoeuver when the helicopter waits for a quiescent state of the ship deck before landing.

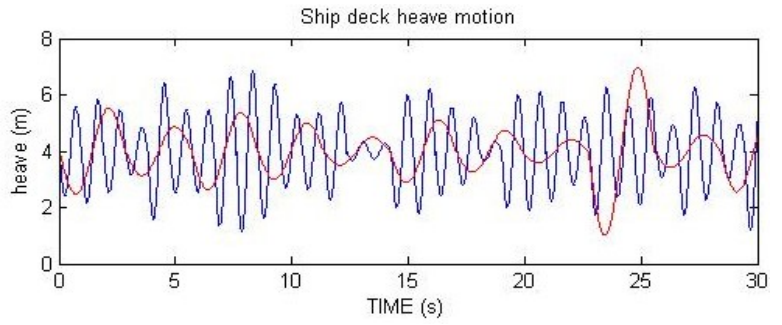

Figure 8. Ship deck motion under sea state level six at 30 knots ship speeds (red) and null ship speed (blue).

\section{Conclusions}

Accurate, yet simple, simulation environments are fundamental tools to develop GNC algorithms.

This paper presented an effective simulation environment to be used specifically for design, preliminary test and software implementation of automatic take-off and landing algorithms on a ship deck.

With reference to rotary-wing applications, an original modelling approach based on both empirical relations and appropriate mathematical formulation has been proposed that still accurately reproduce helicopter and ship transactional motion and ship air wake.

Simulation results demonstrate effectiveness and accuracy of such modelling approach.

The developed simulation environment contributed to reduce design time, risks and costs of automatic takeoff and landing algorithms on a ship deck that were successfully tested in flight.

Future work will be focused on the refinement of the model's parameters (especially for what concern the ship air wake model) based on the analysis of flight data.

\section{References}

G. D. Carico, R. Fang, R. S. Finch, W. P. Geyer Jr., H. W. Krijns, and K. R. Long. Helicopter/Ship Qualification Testing, RTO AGARDograph 300, Flight Test Techniques Series, 22, 2003.

V. Gavrilets. Autonomous Acrobatic Maneuvering of Miniature Helicopters, PhD thesis, MIT, 2003.

L. H. Holthuijsen. Waves in oceanic and coastal waters, Cambridge University Press, 2007. doi:10.1017/CBO9780511618536.

W. Johnson. Helicopter Theory, Dover, New York, 1994.
C. H. Kääriä. Investigating the Impact of Ship Superstructure Aerodynamics on Maritime Helicopter Operations, $\mathrm{PhD}$ thesis, University of Liverpool, 2012.

T. von Kármán. Progress in the Statistical Theory of Turbulence, Proceedings of the National Academy of Sciences, 34 (11): $\quad 530-539, \quad 1948$. doi:10.1073/pnas.34.11.530.

D. Lee. Simulation and Control of a Helicopter Operating in a Ship Airwake, PhD thesis, Pennsylvania State University, 2005.

Z. Li. Path Following with Roll Constraints for Marine Surface Vessels in Wave Fields, $\mathrm{PhD}$ thesis, University of Michigan, 2009.

B. W. McCormick. Aerodynamics, Aeronautics, and Flight Mechanics, John Wiley \& Sons, New York, 1995.

MIL-F-8785C. Military Specification Flying Qualities for Piloted Airplanes, 1991.

G. D. Padfield. The making of helicopter flying qualities: A requirements perspective, The Aeronautical Journal, 102 (1018): 409 - 437, 1998.

G. D. Padfield. Helicopter Flight Dynamics: The Theory and Application of Flying Qualities and Simulation Modeling, AIAA Education Series, Virginia, 1996.

T. Perez. Ship Motion Control, Springer, 2005. doi:10.1007/1 84628-157-1.

M. B. Tishler and R. K. Remple. Aircraft and Rotorcraft System Identification, AIAA Education Series, Virginia, 2006. doi:10.2514/4.868207.

X. Yang, H. R. Pota, and M. Garratt. Design of a GustAttenuation Controller for Landing Operations of Unmanned Autonomous Helicopters, In Proceedings of 18th IEEE International Conference on Control Applications, Saint Petersburg, Russia, 2009. doi:10.1109/CCA.2009.5281074. 